\title{
Component Software Systems for Surface Water \\ Simulation
}

\author{
L. Postma, P.M.A. Boderie, J.A.G van Gils, and J.K.L. van Beq'- \\ WL | Delft Hydraulics, P.O. box 177, \\ $2600 \mathrm{MH}$ Delft, Netherlands \\ \{Leo.Postma, Pascal.Boderie, Jos.vanGils, Jan.vanBeek\} @WLdelft.nl \\ http://ww. WLdelft.nl
}

\begin{abstract}
The simulation of the behaviour of surface water is a complicated activity, dealing with waves, water flow, transport of substances, water quality, ecology and morphology. These processes have their own characteristic time scales from seconds for surface waves through years for ecology and decades for morphology. These processes also have their own spatial scales and requirements for local refinement and solvers. Increasingly more specialised models become available that can describe part of this system. Furthermore, the surface water system is increasingly considered jointly with ground water systems, the atmosphere and human influences from the land. Component Software Technology is a powerful technique to cope with these demands as will be illustrated with the Delft3D system of WL | Delft Hydraulics as example. The design and functioning from the top level down to the detail level will be illustrated going down to the tiny components of the water quality processes.
\end{abstract}

\section{Introduction}

"Components are for composition" is the starting line of the book on Component Software of Clemens Szyperski [1]. He is mainly focussing on the re-use of software components and the market for them. He looks at Java and Component Pascal. He also sees Component Software as the compromise between standard software and custom-made software. The robustness of standard software and the flexibility of custom-made software are combined if such software components could be composed to a custom-made application. It is this feature that is the topic of this contribution. The advantages of Component Software Methodology will be shown at three levels of a complex problem-solving environment for surface water systems as a whole and for its water quality in particular.

The highest level is the level of the stacking and plugging together of whole models of different aspects of the surface water system. Models even made by different institutes, if we can agree to sufficiently general interfacing standards. The parallel processing technologies that are being developed nowadays for High Performance Computing and domain decomposition using multiple instances of one simulation software, can then equally well be applied to multiple instances of different models and even of models from different institutes. 
The intermediate level is that of modules within one simulation model. A module for fine cohesive sediments is needed in a morphology model and in a water quality model as well. Use of the same component increases robustness and cost efficiency. It will be shown how the many different numerical solvers are plugged in as alternative components in a water quality model. Especially for numerical solvers it is very well possible to also use third party components.

The lowest level is the level of tiny software components. The water quality modeller is faced with many substances like temperature, oxygen, nutrients, algae in different species, suspended sediments, several heavy metals and many organic micropollutants. More processes determine the interactions between these substances and even more process constants and variables determine the rates at which the processes act. It will be shown how Component Software technology is used to construct a water quality modelling framework and associated user interface without any content. The content, the substances and processes, are contained in an expandable library of tiny components that have exactly the same interface to plug into the framework and a logical functioning that is fully tabulated.

\section{The Global Component Level}

The surface water is permanently in motion. Waves result from blowing winds or passing ships. Wave models describe the propagation and refraction of surface waves on a small spatial scale on a time scale of seconds or in the frequency domain. Attraction of sun and moon produce long waves with time scales of half a day which result in tidal flows. Gravity forces cause rivers to seek the lowest point. Waves and flows are counteracted by bottom friction. This friction may prevent transported clays and sands from settling, but may also scour the bed and remove sediments to elsewhere. On the time scale of decades up to geological time scale the rivers may meander and seas may silt up to land or get formed by scouring. Light and natural content of dissolved substances in the water make algae grow, the beginning of all life in water. Antropogenic nutrients increase this organic production and antropogenic toxic matter decreases it. Algae grow on seasonal time scales within the year. They are eaten by shellfish or small shrimp that are eaten by bigger fish etc. Shellfish and fish reproduce in one to several years. If you want to assess the environmental impact of the construction of a harbour in a river mouth, you may easily end up having to address all aspects that have been mentioned above.

Traditional monolithic simulation systems for surface water will incorporate several of the aspects mentioned, but generally run on the time scale of the fastest process, which is often the water flow (minutes to hours). They thus have limited value for e.g. morphology, water quality and ecology. If separated into different process components and a "scheduler" to plug the components in, the scheduler and interfaces are able to solve the differences in scale (figure 1).

The differences in scale are not limited to differences in time scale. Near field aspects act on spatial scales of a few kilometres but may get their forcing from 


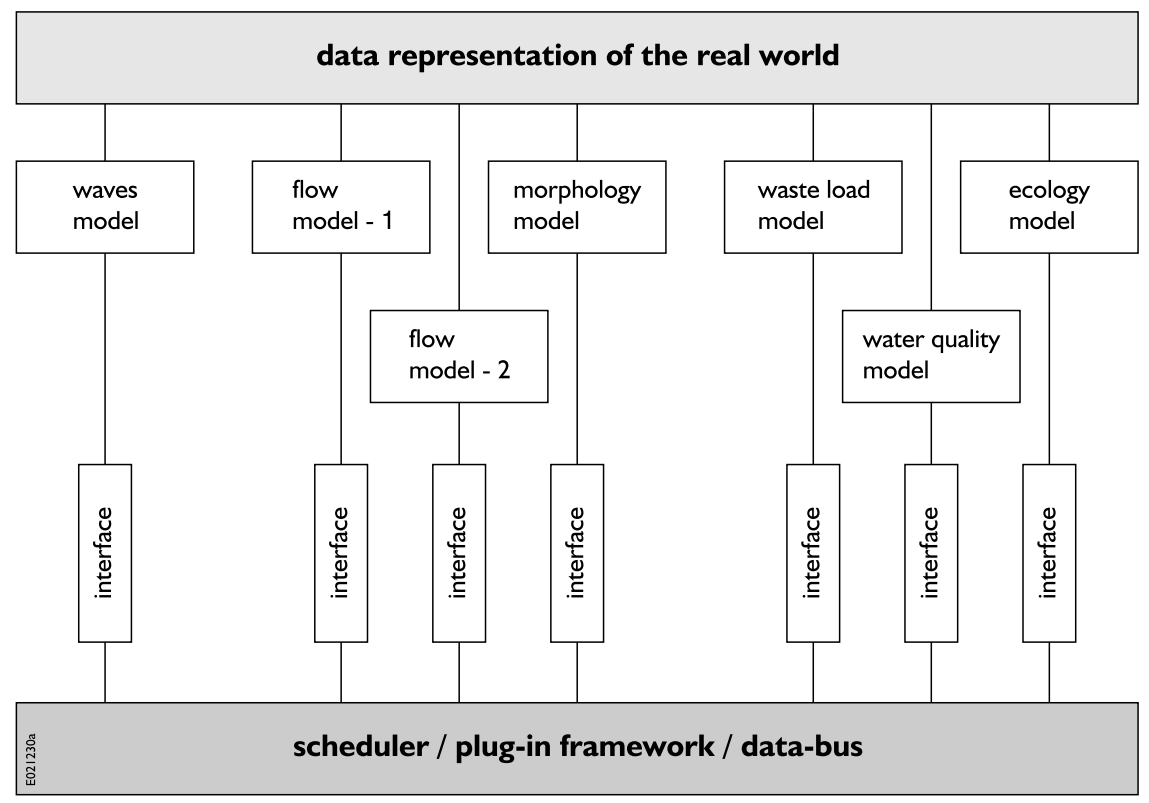

Fig. 1. Scheme of relations between plug-able components in a general surface water simulation system. There is no fundamental difference between using multiple instances of one model for domain decomposition and using different models for different aspects.

oceanic and shelf tidal systems which act on scales of hundreds of kilometres. Generally domain decomposition is used to deal with differences in spatial scale. The area is divided into sub-areas with the required grid sizes. Where the subareas meet an interface takes care of the propagation of the numerical solution of the equations from one domain into the other and backwards. Domain decomposition enhances performance because the executables for each domain can compute in parallel on different processors. This is however too limited a view. The same technique can be used to run models in concert for totally different aspects of the water (e.g. flow and morphology or flow and water quality). This is an important aspect for the user of the system even on single processor systems where no performance boost results from the concurrent use of multiple processors.

In Hong Kong waters [2] a 3-dimensional hydrodynamic model computing a 14 day spring-neap cycle with time steps of one minute was used in combination with a water quality model for full annual cycles at $2 \times 2 \times 2$ aggregated grid cells and time steps of half an hour. In multi year morphological computations the scheduler uses the hydrodynamics model some now and then for a tidal cycle. Meanwhile the bottom elevation is dynamically adjusted by the morphological model [3]. Furthermore the 2 way interface between the waves module and the hydrodynamic module accounts both for the influence of the water currents on the wave pattern and for the wave driven hydrodynamic currents that are so 
essential in near-shore waters [4]. From the Delft1D system (Sobek) the joint simulation of branched $1 \mathrm{D}$ rivers, dike burst and $2 \mathrm{D}$ supercritical inundation flow can be mentioned [5]. Also the joint simulation of partly filled, fully filled and overflowing sewer systems and overland flow through streets towards the river [6] is an example of the benefits of the approach of the use of large plugable components.

\subsection{The Importance of Interfaces}

The careful design of the plugs and interfaces is the issue at stake at this level. Both the components (waves, flow, morphology, water quality and ecology) and the interfaces need to be generic. This means that they should act independent of the application and of the setting of the study and they should act equally well for all supported applications and settings. The maximum number of direct interfaces between $n$ plug-able components that mutually link is $n *(n+1) / 2$ if a component is also allowed to link to itself. A careful design with a scheduler and a common data bus can result in a star shape topology, equivalent to the PCsystem bus, reducing the amount of interfaces to $\mathrm{n}$. The latter is the topology of most of the current Delft3D system, although also some bilateral interfaces exist. A step further is to design the plugs and busses in such a way that new components plug in easily. Such a new component could be a different simulation system for e.g. atmospheric influences or for groundwater flow or for wasteload generation by activities on land. If this happens and standards between different companies and institutes could be derived, then a really open system results. It is this type of standards that is currently aimed at by a number of Dutch institutes cooperating in the OMS (open modelling system) project [7].

The Delft water quality model, which will later on be used as example for lower level component software architecture, shows this openness. It interfaces not only to the Delft3D system for 2- and 3-dimensional modelling but also to the Delft1D (Sobek) system for 1- and 2-dimensional modelling. Interfaces exist with e.g. the HydroQual ECOM model (a further development of the 3D Princeton Ocean hydrodynamics Model POM) [8]. Furthermore joint applications with the Shyfem finite element model for Venice Lagoon [9] and with the CARDINAL model for Neva Bay, St. Petersburg [10] have been reported. The software for all these applications and connections only differs in its interfacing with the water flow module. The water quality simulation part itself was identical. Basic demands for a successful set of interfaces at this level are:

- the objects transported by the interface should have a strict separation between the content of the information and the administration/schematisation

- the content should fully and unambiguously describe the state of the module without omissions and without contradictions, in clear SI units

- the content should obey the relevant basic laws of physics and chemistry (conservation of mass, energy, momentum)

- the administration should be simple in its structure and in its reference to the content of the information 
- the components should run equally well of-line or on-line triggered per time step. They should allow for multiple instances within one application

There are no specific IT demands in here because decisions with respect to message passing or shared memory, separate executables or multi-threading, etc. are basically part of the scheduler. The separation of all content and its administration in the interface and the techniques of invocation and communication into the scheduler makes it possible to create a scheduler that is generic to most connected modules. It also makes it possible to let a non-IT scientist create the interface because it plugs into the scheduler in a simple and well-described way. Some relaxation is allowed if a component interfaces with itself in e.g. a domain decomposition setting. Then very special structures and information flows can be used that allow for the seamless propagation of the computational nucleus from one domain to another. This is no limitation to what is said before if this is a proprietary, hidden interface rather than a public interface.

An important conclusion with respect to User Interfaces can be drawn. If the user interface is not monolithic but structured with exactly the same topology as the plug-able component system, then the same advantages apply for the User Interface. The user interface of a plug-able component plugs into the user interface of the scheduler and infers from it the required information from the GUI of the real world data (generally a GIS). Furthermore the above concept allows all plug-able models and user interface components to use their own programming language and other IT solutions of choice. It even allows for maintenance and modernisation of the system on a per-component base, which is very attractive for large and complicated systems.

\section{Plug-Able Decomposition within a Global Component}

A global simulation component is in itself also modularly structured. Figure 2 gives an overview of the modules that are present in a simulation model in general with specific detail for the water quality part of a water simulation system. The time update and time output module will contain interfaces to the scheduler because the component is allowed to interact on-line with other components during the simulation. The modules within such a global simulation component could also be designed in such a way that they are plug-able and interchangeable. For the Delft Water Quality module this holds for the numerical integrators of the advection-diffusion equation (ADE-solvers). Some 20 solvers are implemented implicit, explicit, ADI, predictor corrector type, etc. Two of the solvers are solvers that are equal to solvers within the Delft3D-Flow module [11]. One of the solvers is the solver that is also used in the Princeton / ECOM model for the advection-diffusion part [8]. These three solvers work on regular (rectilinear or curvilinear) grid topologies. Other solvers work on any stacking of computational elements through a finite volume approach. Although not implemented yet, a finite element solver could easily be implemented as 21 st. The code of the Princeton and Delft3D-Flow solvers have been left unchanged, they were plugged in using an interface. 


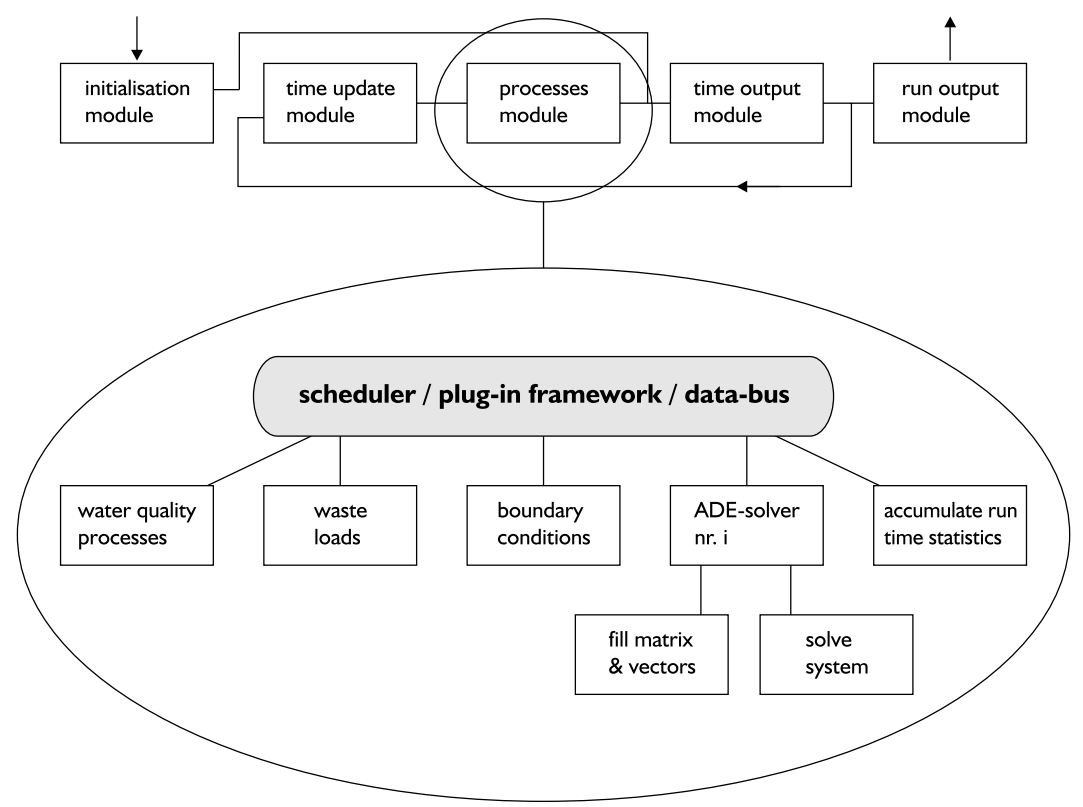

Fig. 2. Plug-able modules within a software component of the complete surface water simulation system further detailed for the water quality component.

The reason for this abundance of solvers is that some are faster but not so accurate, others perform best for problems with steep gradients, others for smooth gradients, some are best for advection dominated problems, others for mixing problems. Instead of having different models, one for each solver, it is much more convenient to have a 'solver-switch', leaving the remainder (waste loads, boundary conditions, water quality processes) unchanged.

The key to this plug-able modularity of solvers is basically the same as to the interfacing between global components discussed in Sect. 2. A thorough separation of functions between modules; a separation of the content of the data and the administration of the data; a clear and consistent interface between the modules and the main program (which is basically an internal scheduler of the time loop of functional modules). The initialisation module initialises the administration in one of two flavours: a regular grid layout for 4 of the solvers or a link node administration for the arbitrarily stacked cells of the other solvers. For a finite element solver just a third type of administration would add. All the other routines and modules of the water quality part like those for processes, waste loads, open boundary conditions and on-line statistics just act on all or several grid cells without knowing whether they are stacked regularly, irregularly or forming finite element triangles. The administration is only added and used in the ADE solvers. The ADE solvers in their turn know nothing about processes, loads and boundaries, that is all condensed in a forcing vector per grid cell. 


\section{Tiny Single Process}

One of the modules of the water quality model is the water quality processes module (figure 2). It could contain the mortality of bacteria that are released with the wastewater, the growth of algae in the water using nutrients, but also the partitioning of heavy metals to suspended silts. Many processes depend on temperature and run faster in warmer water. Some processes depend on the water velocity, like the settling of fine particles, clays and sand from the water column towards the bottom. Some processes are simple like first order reactions, others involve a complete and complicated sub-model like the determination of a chemical equilibrium by minimising Gibbs Free Energy [12].

Traditional water quality modules have these processes programmed in a subroutine. The parameters are provided through the User Interface and/or input file. For any subsequent study application either the same setting of processes is used or a copy is taken and modified depending on the demands of the study (e.g. a certain organic micropollutant added). The problem with these changes is to keep track of all different versions and also the User Interface has to be adapted to provide additional input etc. Any third party user must either dig into extensive and non-trivial code or can only use the model as is.

This urged Delft Hydraulics to develop a generic and expandable concept. We used the approach of plug-able tiny software components for this aim. Each water quality process is a separate component that looks fully identical to the framework where it is plugged in. The 'contract' of the component with the framework specifies the names of the items that it needs as input and the names of the items that it will produce as outputs. The output comes in two flavours: one is that of entities that can be used by other process components and the other is that of "fluxes of mass". The last product is the process flux from one substance to another. These fluxes are the arrows in the commonly used flow charts of water quality models.

For each state variable (called 'substance') a table defines which fluxes act on it and with which stoichiometry. The nitrification flux for instance has stoichiometry -1.0 for Ammonia that is removed by bacteria. It has +1.0 for Nitrate that is formed to the same rate. It also has -4.57 for Oxygen because the flux uses so many gram of Oxygen per gram of Nitrogen. For each flux a table defines which plug-able component is used to compute it and what it has to use as input and what the names of its output variables are. This mapping of components allows to use the same component for e.g. the partitioning of all heavy metals.

For the input (e.g. a reaction coefficient) default values may be specified. If a certain component needs the 'Temperature' as input then either the default value is used (if there is one), or a user defined value (or spatial field of values) is used, or the model result with this name is used if 'Temperature' is modelled itself. The framework takes care that the component automatically receives its input and that the output is delivered where needed in the model. This has far reaching implications. If an input of one process has to be provided by another process, then this other process is switched on automatically to supply the necessary information. 


\subsection{Specialist Use}

A specialist who wants to add e.g. a new organic micropollutant to the library of state-variables and components adds the name of this pollutant to the list of supported substances. He specifies the fluxes that act on it (e.g. volatilisation at the surface, decomposition under influence of UV-radiation and partitioning to living and dead organic matter). For each of these fluxes he specifies the name of the software component that computes it, what the names of the input and output variables are and whether there are default values for certain inputs. In the situation that a process formula is not present in the library, a new software component is written in a high level language like Fortran-95 with simple and clear calling conventions. The component only contains the missing formula.

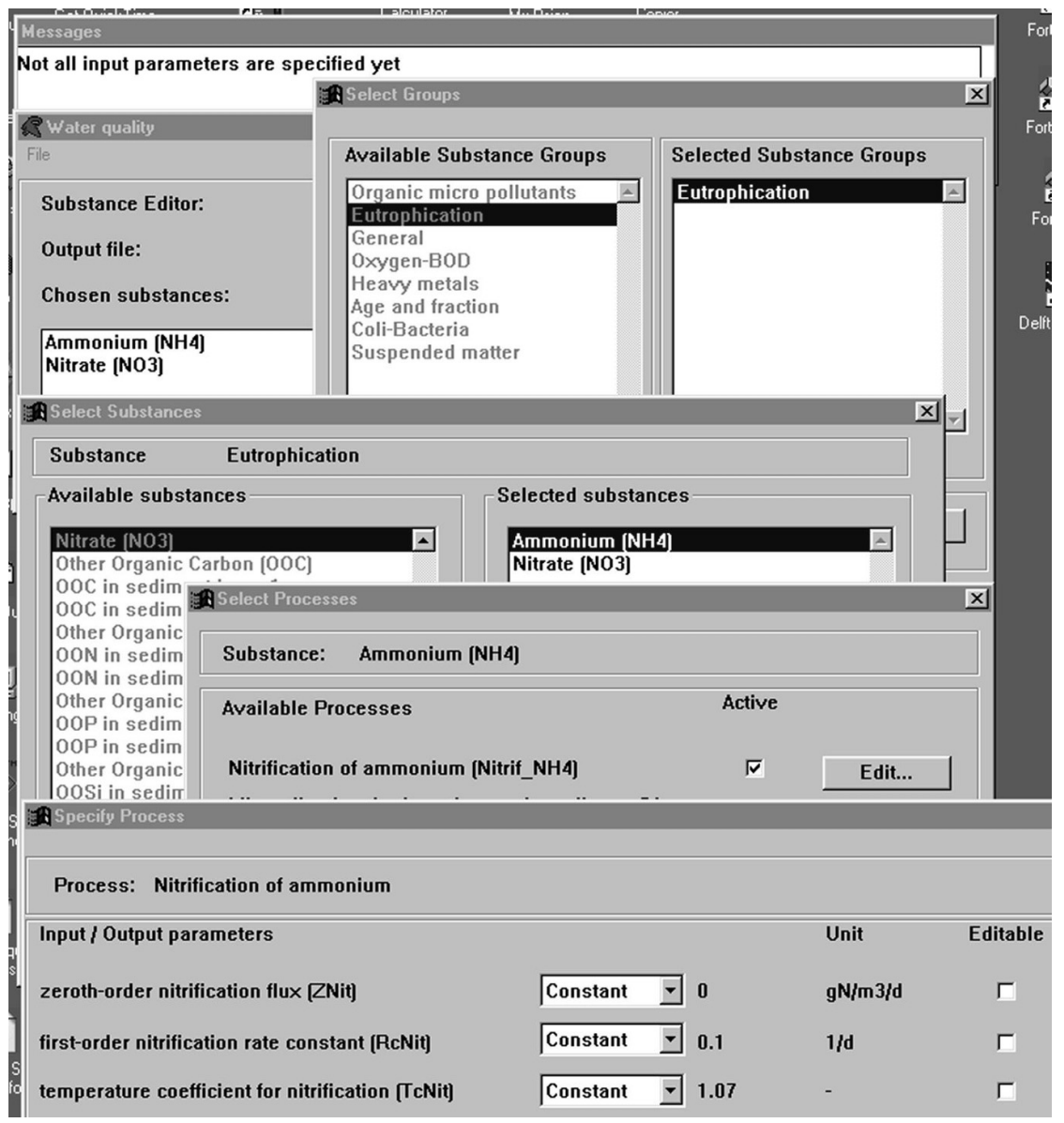

Fig. 3. First five levels of the hyperlink system to select substances, processes and items to be editable by the end user. The 6 th level is in the water quality component user interface. 
In this way at present already over 200 substances are supported, using over 1000 constants, parameters and other information for their processes. The processes are computed by some 150 tiny plug-able components. The tables that define the logic of this whole system are also used to fill the user interface. This means that the specialist who added the new organic micropollutant will notice that his information is automatically contained in the GUI.

\subsection{Use by a Modeller and an End-User}

How can a water quality modeller use this all? A 6 level deep hyperlink system is provided (figure 3). Level one is the shopping basket. It contains everything that is selected for the model. Level 2 consists at present of 8 substances groups. By selecting a group, level 3 is entered to select substances. Substances from different groups can be selected jointly. Once a substance is selected, level 4 is entered by clicking it and the fluxes that act on the substance are shown. In level 5 the input to the tiny components computing a selected flux is editted and variables are made 'user-editable'. The chosen setting of the first 5 levels is saved and exported to level 6 , which is outside of this hyperlink system. Level 6 is in the User Interface of the water quality model itself. There the specification of the input takes place. Saved results of level 5 can be selected by the end-user as 'the water quality model' for a certain project. The end-user only sees the items that were specified to be user-editable.

The entire over 1000 items in the processes system can be selected for output. Nobody will do so at one time but the selection of certain intermediate results or derived properties makes it easy to monitor why certain processes act as they do. Special mentioning deserves the group of statistics plug-in components. They allow the derivation of time averages, spatial averages, exceedence frequency etc. of any modelled entity. This is important when determining whether water quality objectives are violated.

The whole procedure delivers a water quality model that is as lean or fat as specified in the first 5 levels. One conservative substance gives a very small and fast running model. Full eutrophication and toxic substances modelling will become larger and slower.

\section{Conclusions}

The concept of Component Software has major advantages over traditional software architecture also for systems for the simulation of surface water. The Delft3D-Delft1D case shows that this technique is feasible and to a certain extent already proven technology. At the high level of whole models this approach can resolve the problem of scales in time, space and process settings. At the intermediate level interchangeable modules like turbulence modules and ADE solvers can be used in this way. At the level of tiny process components generic and expandable libraries can be made and systems of compicated mutual interactions can be formed from it. 
The interfaces are of equally profound importance for the small and large components. They are the contracts between the user and the provider of the components. If the future is in Component Software, and we believe it is, then the future is in people that are able to provide generic and useful interfaces. They must be able to combine experience in software development with insight in and knowledge of the application field.

\section{References}

1. Szyperski, C.: Component Software. Beyond Object-Oriented Programming. Addison-Wesley, ACM Press New York (1998)

2. Postma, L., Stelling, G.S., Boon, J.: 3-dimensional Water Quality and Hydrodynamic Modelling in Hong Kong III. Stratification and Water Quality. In: Lee, J.H.W., Jayawardena, A.W., Wang, Z.Y. (eds.): Environmental Hydraulics. A.A.Balkema, Rotterdam Brookfield (1999)

3. Roelvink, J.A., Boutmy, A., Stam, J.-M.: A simple method to predict long-term morphological changes. In: Edge, B.L. (ed.): Proc. Coastal Engineering, Copenhagen, Denmark (1998) 3224-3237

4. Morris, B., Thornton, E.B., Reniers, A.: Nearshore wave and current predictions compared with field observations. In: Proc. Coastal Dynamics '01 Conference, Lund, Sweden (2001) 788-797.

5. Verwey, A.: Latest Developments in Floodplain Modelling - 1D/2D Integration. Keynote lecture 6th Conf. On Hydraulics in Civil Engeneering, Hobart, Australia (2001)

6. Heeringen, K.J. van, Verwey, A., Melger, E.: Dutch Approach to High Speed Urban Drainage Modelling with SOBEK. In: Proc. 9th Int. Conf. On Urban Storm Drainage, Portland, USA (2002)

7. Hummel, S., The, B.S.T., Branchett, S.E., Brinkman, R.: DelftIO, standardised application communication. In: Proc. 5th Int. Conf. on HydroInformatics, Cardiff, UK (2002)

8. Blumberg, A.F., Oey, L.Y.: Modelling Circulation and Mixing in Estuaries and Coastal Oceans. Adv. In Geophysics, 28A (1985) 525-547

9. Runca, E., Bernstein, A., Postma, L., Di Silvio, G.: Control of macroalgae blooms in the Lagoon of Venice. In: Ocean \& Coastal Management, 30 No 2-3 (1996) 235-257

10. Boon, J.G., Klevanny, K.A., Matveyev G.V., Pagee, H. van: Coupling of CARDINAL and DELWAQ modelling systems for water quality simulations in the Neva Bay. Proc. Of the int. conf. on Ecology and development of the North-West region of Russia. St. Petersburg-Kronschtadt, Russia (1997) 319-320

11. Stelling, G.S., Kester, J.A.Th.M.: On the approximation of horizontal gradients in sigma co-ordinates for bathymetry with steep bottom slopes. Int. J. Num. Meth. Fluids, 18 (1994) 915-955

12. De Rooij, N.M., Smits, J.G.C.: Mathematical Modelling for Assessment of Soil Pollution. In: Salomons, W., Förstner, U., Mader, P. (eds.): Heavy Metals, Problems and Solutions. Springer Verlag, Berlin (1995) 